\title{
Reply to a letter to the Editor regarding the article "Testosterone attenuates hypoxia-induced hypertension by affecting NRF1- mediated transcriptional regulation of ET-1 and ACE"
}

\author{
Shan Jiang ${ }^{1,2} \cdot$ Guijuan Chen ${ }^{1} \cdot$ Zhihui Yang $^{1} \cdot$ Dan Wang ${ }^{1} \cdot$ Yapeng Lu $^{1} \cdot$ Li Zhu $^{1,3} \cdot$ Xueting Wang $^{1}$
}

Received: 23 August 2021 / Accepted: 30 August 2021 / Published online: 27 September 2021

(c) The Japanese Society of Hypertension 2021

\section{Dear Editor-in-Chief,}

We would like to thank Dr. Martin, Gregoire and Johannes for their pertinent concern about the effects of testosterone in mice and humans under hypoxic conditions. Hypoxia causes an increase in blood pressure in males. Our research also suggested that the blood pressure of male rats increased markedly after hypoxia exposure [1]. We agree that blood pressure in men is more sensitive to hypoxia. As Martin et al. described, the regulation of blood pressure is influenced by age, the sympathetic nervous system, the respiratory system, the cardiovascular system, sex, and other factors [2]. Sex hormones are only one aspect. It is widely known that female sex hormones, such as estradiol, play a protective role in blood pressure regulation [3]. Here, we aim to explain the mechanism underlying the sex differences in blood pressure changes after hypoxia exposure. We found that testosterone protects male mice from systemic blood pressure elevation. However, our early research found that hypoxia significantly reduced testosterone levels, leading to reduced blood pressure protection $[4,5]$. This finding indicates that blood pressure changes more obviously in men than in women after hypoxia exposure. Of course, as Martin and colleagues suggested, the multiple effects of testosterone and the sex differences in blood pressure regulation deserve more in-depth study.

\section{Compliance with ethical standards}

Conflict of interest The authors declare no competing interests.

Publisher's note Springer Nature remains neutral with regard to jurisdictional claims in published maps and institutional affiliations.

\section{References}

1. Jiang S, Chen G, Yang Z, Wang D, Lu Y, Zhu L, et al. Testosterone attenuates hypoxia-induced hypertension by affecting NRF1mediated transcriptional regulation of ET-1 and ACE. Hypertens Res. 2021; https://doi.org/10.1038/s41440-021-00703-4.

2. Hogarth AJ, Mackintosh AF, Mary DA. The effect of gender on the sympathetic nerve hyperactivity of essential hypertension. J Hum Hypertens. 2007;21:239-45.

3. Ashraf MS, Vongpatanasin W. Estrogen and hypertension. Curr Hypertens Rep. 2006;8:368-76.

4. Wang X, Jin L, Jiang S, Wang D, Lu Y, Zhu L. Transcription regulation of NRF1 on StAR reduces testosterone synthesis in hypoxemic murine. J Steroid Biochem Mol Biol. 2019;191:105370.

5. Wang X, Zou Z, Yang Z, Jiang S, Lu Y, Wang D, et al. HIF 1 inhibits StAR transcription and testosterone synthesis in murine Leydig cells. J Mol Endocrinol. 2019;62:1-13.

\author{
Li Zhu \\ zhulizhou@ntu.edu.cn \\ $\triangle$ Xueting Wang \\ wangxueting@ntu.edu.cn \\ 1 Institute of Special Environmental Medicine, Nantong University, \\ Nantong, China
}

2 School of Life Science and Technology, China Pharmaceutical University, Nanjing, China

3 Co-Innovation Center of Neuroregeneration, Jiangsu Key Laboratory of Neuroregeneration, Nantong University, Nantong, China 